Quelles perspectives pour l'Asie?

\title{
Perspectives de croissance et de développement de l'éducation en Asie
}

Prospects for the growth and development of education in Asia

Perspectivas de crecimiento y de desarrollo de la educación en Asia

\section{Rangachar Govinda}

Traducteur : Jérôme Quintana

\section{OpenEdition Journals}

Édition électronique

URL : https://journals.openedition.org/ries/4371

DOI : 10.4000/ries.4371

ISSN : 2261-4265

Éditeur

France Education international

Édition imprimée

Date de publication : 4 avril 2015

Pagination : 141-150

ISBN : 9782854206067

ISSN : $1254-4590$

\section{Référence électronique}

Rangachar Govinda, «Perspectives de croissance et de développement de l'éducation en Asie », Revue internationale d'éducation de Sèvres [En ligne], 68 | avril 2015, mis en ligne le 04 avril 2017, consulté le 02 juillet 2021. URL : http://journals.openedition.org/ries/4371 ; DOI : https://doi.org/10.4000/ries. 4371

(c) Tous droits réservés 


\title{
Perspectives de croissance et de développement de l'éducation en Asie*
}

\author{
Rangachar Govinda \\ National University \\ of Educational Planning \\ and Administration, \\ Inde
}

Un colloque sur l'éducation en $A i^{1}$ se serait-il tenu il y a 25 ans à Paris? Qu'est-ce qui déclenche aujourd'hui le besoin d'évoquer cette question? On pourrait trouver de nombreux facteurs déclencheurs, mais la montée de l'Asie sur un plan économique en est incontestablement un, et pas le moindre. De nombreuses personnes pensent et écrivent que le XXI ${ }^{\mathrm{e}}$ siècle appartient à l'Asie. Un rapport de la Banque asiatique de développement (Asian Development Bank : ADB) publié en 2010 s'intitulait Asia 2050, realizing the Asian century (L'Asie en 2050 : la réalisation du siècle de l'Asie). Les attentes à l'égard de l'Asie sont élevées, à l'intérieur comme à l'extérieur du continent asiatique. Il ne s'agit pas d'une simple aspiration : les gens ont le sentiment que la montée de l'Asie est bel et bien possible.

Parler de la montée de l'Asie, c'est pointer un paradoxe. L'Asie fut le berceau de civilisations anciennes et abrite aujourd'hui les pays connaissant la plus forte expansion économique au monde, ainsi que les trois plus grandes économies de la planète. On la considère également comme le tremplin de la révolution des technologies de l'information et de la communication (TIC), la Chine fournissant le matériel informatique et l'Inde les logiciels. Cette montée est néanmoins paradoxale car l'Asie possède par ailleurs le plus grand nombre d'analphabètes et d'enfants déscolarisés au monde. Selon moi, c'est cela qui détermine ce qu'il «faudrait » faire advenir dans le domaine de l'éducation, non pas au sens prescriptif de ce verbe, mais comme un but à atteindre.

Si l'on entend beaucoup parler en Europe de la Chine, de l'Asie du Sud-Est et de l'Asie de l'Est, en règle générale, le point de vue de l'Inde et du sous-continent sud-asiatique est moins représenté. C'est celui que j'adopte aujourd'hui.

\footnotetext{
* Article traduit par Jérôme Quintana.

1. Cet article est issu d'une communication présentée par l'auteur le 14 juin 2014 au Centre international d'études pédagogiques (CIEP), à Sèvres, lors du colloque international «L'éducation en Asie en 2014 : quels enjeux mondiaux ? " (12-14 juin 2014), organisé à l'occasion du $20^{\mathrm{e}}$ anniversaire de la Revue internationale d'éducation de Sèvres. Pour en savoir plus : http://ries.revues.org/3710/ (NdIR)
} 
Je commence par m'interroger sur ce que c'est, l'Asie. Il n'existe pas une seule et unique Asie, un seul et unique miracle, qui nous permettraient de parler de l'Asie dans son ensemble. L'Asie, c'est une cacophonie de façons d'être et de langues, représentant une grande diversité en termes de religion, de langue, de culture, d'héritage historique des différentes régions de l'Asie, de structure politique, de situation actuelle, de contexte de développement. Tout est différent, et pourtant, les gens parlent de "l'Asie». Mais de quelle "Asie» parlons-nous exactement?

Sur un plan historique, il existe de nombreuses manières d'évoquer l'Asie. Autrefois, les évocations concernaient surtout la distinction entre Occident et Orient, et faisaient le plus souvent allusion aux anciennes civilisations de l'Asie. Elles avaient trait aussi au passé colonial ou encore à la nature exotique des pays et des peuples de l'Asie. Aujourd'hui, on évoque l'Asie de manière très différente. Le rapport de l'ADB caractérise ainsi ce changement:

L'Asie est en pleine mutation. Il s'agit d'une mutation véritablement historique. Si elle continue à se développer sur sa trajectoire récente, elle pourrait d'ici 2050 représenter plus de la moitié du PIB mondial, du commerce et de l'investissement, et jouir d'une abondante richesse. Son revenu par habitant pourrait être multiplié par six.

Elle est ainsi porteuse d'une promesse, celle de permettre à quelque 3 milliards d'Asiatiques, aujourd'hui considérés comme pauvres et défavorisés, d'accéder au statut de "riches » selon les critères actuels. En doublant quasiment sa part du PIB mondial, la faisant passer de $27 \%$ en 2010 à $51 \%$ d'ici 2050, l'Asie retrouverait la position économique dominante qu'elle occupait à l'échelle mondiale il y a environ 250 ans, avant la révolution industrielle. Certains parlent ainsi de «siècle de l'Asie ». Mais le rapport se veut naturellement prudent :

Si cette issue prometteuse est plausible, elle n'implique pas pour autant que, dans son ascension, l'Asie connaîtra un destin tout tracé. Cela pourrait ne pas être le cas.

Le rapport indique ensuite que " cette possibilité d'ascension de l'Asie est assortie d'un certain nombre de conditions et de mises en garde ». Quelles sont-elles?

Partant de l'idée que l'unité de l'Asie ne s'entend qu'au sens géographique du terme, comment cette montée de l'Asie peut-elle advenir ? Par ailleurs, si l'éducation doit être au cœur de cette mutation de l'Asie, comment celle-ci doit-elle s'opérer ? Que le XXI ${ }^{\mathrm{e}}$ siècle appartienne ou non à l'Asie, il nous faut répondre à un certain nombre d'autres questions. Les établissements scolaires en Asie doivent être au cœur de ce développement imaginé, et si tel est le cas, sont-ils prêts à assumer cela ? Sont-ils suffisamment équipés ? Quelles leçons du passé peut-on tirer des différents pays de l'Asie? Et quelles pourraient être les 
trajectoires et les perspectives de croissance et de développement de l'éducation en Asie ? Ces pays ont-ils emprunté la bonne direction pour faire face aux défis du $\mathrm{XXI}^{\mathrm{e}}$ siècle qui s'annoncent?

\section{QUELQUES CARACTÉRISTIQUES PROPRES À L'ASIE EN MATIÈRE DE DÉVELOPPEMENT}

Dans cette réalité qui diffère grandement d'une région à l'autre de l'Asie, certains pays doivent-ils prendre les rênes pour transformer l'éducation ou le développement se fera-t-il en Asie de manière générale? Quels types de transformation doivent-ils avoir lieu dans ces pays en particulier et en Asie plus généralement ? Ces pays seront-ils les initiateurs du changement et de l'innovation ou bien se contenteront-ils de s'adapter aux changements venus du monde extérieur ou de les assimiler, comme on a pu l'observer ces dernières années ?

L'Asie tente de s'imposer dans un monde très différent de celui qui s'est imposé, à savoir le monde occidental, européen et américain, qui a connu une importante ascension lors de la révolution industrielle. Mais il nous faut reconnaître deux différences importantes. Premièrement, l'Occident s'est développé de manière lente, parallèlement à la révolution industrielle, alors que l'Asie tente d'accélérer le pas. Comme le rappelle une commission culturelle de l'Unesco :

[...] aucune génération dans l'histoire de l'humanité n'a été confrontée à une transformation aussi rapide et soutenue que la présente génération. Ces changements rapides affectent de manière profonde les conditions de vie des individus, quel que soit leur lieu de résidence, et il faut prendre cela en compte si les enfants et les jeunes d'aujourd'hui doivent participer de manière pleine et entière au monde de demain.

Deuxièmement, l'Asie tente de s'imposer dans un contexte mondial différent de l'époque relative à la montée de l'Occident. Trois facteurs, qui s'entrecroisent à l'échelle mondiale, semblent définir le contexte du développement général et éducatif de l'Asie, à savoir : la mondialisation, les inégalités croissantes et les technologies de l'information et de la communication. Tout le monde écrit à propos de la mondialisation, mais selon les auteurs, on n'entend pas les choses de la même façon. Même si l'on prend en compte le sens fondamental de l'expansion de l'économie de marché, on doit redéfinir les contours du discours sur l'économie de marché. Si Adam Smith écrivait aujourd'hui La Richesse des nations, l'écrirait-il de la même manière? Traiterait-il des mêmes questions aujourd'hui, dans ce contexte actuel de mondialisation?

Le deuxième facteur, comme l'a indiqué Amartya Sen, est le fait que nous vivons dans un monde qui n'a jamais connu autant d'opulence, une opulence qu'il aurait été même difficile de concevoir il y a un siècle ou deux. Pourtant, nous vivons également dans un monde où sévissent une remarquable pauvreté, un remarquable dénuement et une tout aussi remarquable oppression. 
Il existe de nombreux problèmes nouveaux, mais aussi d'anciens problèmes, dont la persistance de la pauvreté et la non satisfaction de besoins élémentaires. ${ }^{2}$ Le fait de vaincre ces problèmes constitue une part importante de l'exercice du développement. Alors que les journaux regorgent d'articles sur les personnalités au succès retentissant et aux réseaux très influents en Asie, la grande pauvreté, paradoxalement, est aujourd'hui profondément enracinée en Asie. La lutte contre les inégalités doit se faire parallèlement à la lutte en faveur de l'éducation.

Le troisième facteur est l'émergence d'une nouvelle fracture numérique dans la société de la connaissance, qui se trouve être particulièrement profonde en Inde. De vastes changements économiques, sociaux et culturels s'opèrent grâce aux nouvelles technologies numériques, en pleine évolution, et aux infrastructures qui les accompagnent. Prenons par exemple les plateformes de réseaux sociaux : il y a seulement quelques années, celles-ci n'existaient pas, mais aujourd'hui, elles peuvent donner naissance ou mettre fin à un gouvernement ou encore définir pour de nombreux jeunes le programme de la soirée à venir. Il s'agit d'un monde qui s'est développé à toute vitesse, exerçant un contrôle non seulement sur notre vie politique mais aussi, pour beaucoup d'entre nous, sur notre vie sociale. En effet, d'une part, les transformations technologiques interviennent à un rythme sans précédent, entraînant de fait une compression du temps et de l'espace, et, d'autre part, la connexion au réseau internet implique la juxtaposition de diverses géographies, langues et idéologies. La réalité contemporaine s'en trouve radicalement changée et exige de repenser notre façon de vivre, de travailler et d'apprendre. Je pense que c'est vraiment cela qui détermine la raison pour laquelle nous devons repenser toute l'éducation.

Tout le monde semble voir l'éducation comme un moyen stratégique de faire face aux conséquences de ce contexte de mondialisation, d'inégalité et de révolution liée aux TIC, qui évolue très vite. Mais, concrètement, qui va prendre cela en charge ? Qui va prendre la tête de tout ce mouvement pour transformer le contexte éducatif en Asie?

\section{ÉLÉMENTS DE CONTEXTE HISTORIQUE}

Bien que le développement en Asie se décline de façon très diverse selon les pays, un bref rappel de l'histoire des cinquante ou soixante dernières années est peut-être à même d'éclairer notre compréhension des changements qui s'opèrent actuellement dans le domaine de l'éducation. Alors que le monde sortait de la Deuxième Guerre mondiale et de la période de colonisation, au sein de l'Asie, une seule étoile brillait au firmament : le Japon. Pendant plus d'un quart de siècle, on ne fit guère attention aux autres pays de la région. Pour la génération de ceux qui ont grandi dans les années 1950 et 1960, tout ce dont on nous parlait

2. Certains, comme Zygmunt Bauman, considèrent que les inégalités croissantes sont des « dommages collatéraux inévitables de l'ère de la mondialisation ». Je ne suis pas sûr qu’il faille adhérer à ce type de raisonnement. 
pendant ces 25 ans, c'était l'histoire de l'Inde vue par les Chinois et, encore et toujours, l'exemple du Japon et de son ascension. Puis vinrent les Tigres de l'Asie de l'Est, qui connurent une mutation rapide et remarquable, notamment la Corée du Sud, qui se distingua particulièrement, rejoignant le club élitiste des pays industrialisés. Alors que tout le monde se demandait ce qui pourrait advenir, au-delà de l'exemple du Japon, d'autres pays émergèrent en très peu de temps. Que s'est-il passé durant les deux dernières décennies? La Chine et l'Inde ont incontestablement accédé au rang de grandes puissances économiques. Ces deux pays, qui recevaient il y a trois ans des aides au développement, financent aujourd'hui cette aide. Trois pays de la région (le Japon, l'Inde et la Chine) figurent parmi les quatre plus grandes économies de la planète. Les données démographiques sont également en faveur de la Chine et de l'Inde, en termes de population active. Elles regroupent à elles deux plus du tiers de la population mondiale et possèdent le plus grand réservoir de jeunes talents qui contribuent à leur croissance et à leur développement économique. Il va de soi que la Chine et l'Inde devront également être le principal vecteur de transformation de l'éducation en Asie. Cela ne veut pas dire que nous ne devons pas reconnaître le développement, à la fois sur le plan de l'économie et de l'éducation, des autres pays de la région, tels que Singapour, la Thaïlande, la Malaisie et l'Indonésie.

Cependant, la Chine et l'Inde portent aussi un fardeau. Si la croissance économique faiblit dans ces deux pays (selon certains économistes, la Chine et l'Inde pourraient tomber dans le piège du revenu intermédiaire et stagner), cela n'augurera rien de bon pour l'ensemble de l'Asie. De ce fait, la montée de l'Asie est étroitement liée à la situation de l'Inde et de la Chine, tant sur le plan de l'économie que sur celui de l'éducation. Comme l'indiquait déjà le rapport de l'ADB, la condition essentielle de notre réussite dépend de la qualité de l'éducation que nous proposons. Cela implique à mon avis que la Chine et l'Inde doivent occuper le devant de la scène, sur le plan de la transformation de l'éducation qui doit s'opérer.

\section{OÙ EN EST LE DÉVELOPPEMENT DE L'ÉduCATION EN Asie?}

Ces dernières années, il y a incontestablement eu des progrès considérables en termes d'accès à l'éducation de base pour tous les enfants. Quasiment tous les pays de la région ont pu rendre cet accès possible. Toutefois, les pays ont de plus en plus conscience du fait que, si l'accès à l'enseignement primaire pour tous peut raisonnablement garantir une amélioration générale du taux d'alphabétisation et du développement humain, il est néanmoins peu probable que ces pays parviennent à réduire de façon importante la pauvreté et à créer une société plus équitable, à moins de s'attacher rapidement à améliorer l'enseignement secondaire et supérieur. Si l'on considère que l'enseignement primaire joue un rôle essentiel dans le fait de favoriser la participation des individus et leur accès à la liberté, également dans le fait de leur permettre de vivre leur vie 
dans la dignité et non plus dans la privation de biens fondamentaux, l'enseignement secondaire et supérieur, de son côté, contribue à la prospérité, à transformer l'économie et à établir une société socialement juste. Il ouvre les portes du monde du travail aux jeunes des pays concernés et contribue au développement socio-économique de la collectivité.

Il est très intéressant de constater que, si la région s'inscrit dans une longue tradition en matière d'éducation, le développement global de l'éducation est toutefois loin d'être satisfaisant. Il va de soi que si l'éducation doit jouer un rôle-clé pour permettre à l'Asie d'atteindre le sommet, le système doit radicalement transformer ses caractéristiques fondamentales et s'adapter aux exigences de la nouvelle économie du savoir. L'émergence du secteur privé (qui joue un puissant rôle d'arbitre dans l'accès à l'éducation), notamment en Inde et dans d'autres pays asiatiques, soulève un certain nombre de questions en termes d'équité et de qualité. Je citerai à nouveau le rapport de l'ADB :

À l'avenir, les économies convergentes de l'Asie, et notamment la Chine et l'Inde, ne doivent plus se contenter de rattraper les autres pays, mais doivent être des pionnières, à la pointe de l'esprit d'entreprise et de l'innovation, afin de créer de réelles avancées dans le domaine de la science et de la technologie, rejoignant ainsi le Japon, la république de Corée et d'autres économies à fort revenu. Un domaine particulièrement fructueux, dans lequel la Chine et l'Inde ont déjà enregistré de remarquables succès, est "l'innovation frugale", pour répondre aux besoins de millions d'individus aux revenus modestes, ceux qui appartiennent à ce qu'on appelle le "bas de la pyramide". La condition essentielle (qui fait défaut à de nombreux pays de l'Asie), c'est l'accès à l'éducation de qualité à tous les niveaux, favorisant la créativité et soutenue par un écosystème nécessaire à l'innovation et à l'esprit d'entreprise.

Dans le domaine de l'éducation, le Japon continue d'être en haut de l'échelle, que ce soit sur le plan de la recherche et de l'innovation, de la productivité, ou encore de la représentation dans les indices internationaux de citations de publications. Proche du Japon, la Corée du Sud a incontestablement acquis une position importante en dépassant les autres membres du club des Tigres de l'Asie de l'Est. Sa contribution à la recherche et au développement industriels s'est régulièrement accrue au fil des ans. Si certaines publicités concernant l'enseignement supérieur semblent indiquer que Singapour et Hong-Kong sont incontournables dans ce domaine, ces deux pays sont de petites cités-États, peu susceptibles d'alimenter l'énorme processus de croissance de la région. La Chine et l'Inde ont toutes deux commencé à renforcer l'enseignement secondaire et supérieur mais il reste encore beaucoup à faire. Je crois que dans la région, comme l'indique l'ADB, le poids de la réussite devra être assumé par l'Asie du Sud, qui représentera $40 \%$ de la population de l'ensemble de l'Asie d'ici 2050. Aussi, sans l'Inde et l'Asie du Sud, il sera très difficile de garder ce rythme de développement éducatif et économique en Asie. 


\section{LES CHANGEMENTS NÉCESSAIRES EN ASIE}

Le premier point, c'est que nous avons besoin de modifier notre mode d'engagement auprès des élèves. Gwang-jo Kim a parlé de "changement de l'apprentissage $»^{3}$. Ce que nous avons, c'est une structure créée, ou du moins perfectionnée à l'ère de la révolution industrielle, structure connue depuis longtemps pour être fondée sur le par cour et la mémorisation, sans encourager la réflexion critique, la créativité et la prise de risque, autant de compétences nécessaires pour que chaque pays puisse produire ses propres entrepreneurs et ses innovateurs. Que nous soyons d'accord ou non avec cette généralisation, nous devons aider les élèves non pas à trouver les bonnes réponses mais à développer des solutions créatives, ainsi que la communication et la réflexion critique. Le développement de l'enseignement secondaire et supérieur implique qu'une réflexion accrue doit s'engager : quelle position adoptons-nous dans les relations entre professeurs et élèves/étudiants, et dans la façon dont les transactions éducatives doivent s'effectuer dans nos classes et nos villes? Il est temps que nous réfléchissions à des stratégies plurielles et multifonctionnelles afin d'améliorer réellement la qualité de l'enseignement dispensé à nos enfants.

Le deuxième point que je voudrais souligner, c'est que l'Asie doit remporter la course entre l'éducation et la technologie. C'est le titre d'un livre récent de Claudia Goldin et Lawrence Katz (2008), deux auteurs américains, selon lesquels le changement technologique, l'éducation et les inégalités participent à une sorte de course. À partir de leur analyse du contexte historique américain, ils formulent la thèse selon laquelle le ralentissement de l'éducation, d'une part, et le développement de la technologie, d'autre part, s'accompagnent de l'accroissement des inégalités. Les pays asiatiques devraient réfléchir attentivement à ce phénomène, il me semble : devant l'accroissement des inégalités, l'élite intellectuelle en Asie doit parvenir à maîtriser cette course entre l'éducation et la technologie, afin d'avancer.

Il existe un second aspect dans cette course entre l'éducation et la technologie, c'est la course entre la création du savoir et l'utilisation du savoir. Aujourd'hui, le rythme auquel le savoir est intégré pour créer de nouvelles technologies est ahurissant. Mais avec le transfert de la recherche de pointe vers l'entreprise privée et le régime actuellement en place de droit à la propriété intellectuelle, c'est devenu un phénomène sélectif, comme le montre le conflit actuel dans le monde des produits pharmaceutiques. En réaction, la demande de partage du savoir s'est accrue, plutôt que d'emmagasiner le savoir à des fins de profit. L'élite intellectuelle d'Asie posera-t-elle la question de la valeur commerciale du savoir? 
Le troisième aspect de cette course entre l'éducation et la technologie concerne bien sûr le secteur même de l'éducation, en termes de capacité des établissements d'enseignement à incorporer et intégrer les TIC dans le processus d'enseignement et d'apprentissage. Notre conception traditionnelle de l'enseignement et de l'apprentissage comme processus synchrones laisse aujourd'hui place à une vision de l'enseignement et de l'apprentissage en tant que processus asynchrones, qui n'ont pas à se dérouler de façon simultanée. Étant donné que le plus grand nombre de personnes auxquelles il faut proposer un enseignement se trouve aujourd'hui en Asie, peut-on ignorer ce développement ? Sommes-nous suffisamment impliqués dans les nouvelles technologies? Hélas, l'intégration des nouvelles technologies dans la classe est encore trop faible dans de nombreux pays. Certains relient cela à la vision traditionnelle de la technologie, où celle-ci est considérée comme exogène au processus d'enseignement / apprentissage centré sur le "gourou ", la figure du professeur ou du maître. À mon avis, c'est essentiellement là que réside la faille. Nous ferions bien d'explorer, et non de rejeter, l'hypothèse que l'internet change profondément notre mode et notre processus de réflexion. La façon dont le système éducatif et les établissements d'enseignement en Asie négocieront cette course entre l'éducation et la technologie déterminera la future trajectoire de croissance, ainsi que la place du continent asiatique dans la communauté mondiale.

De plus, l'Asie se caractérise par le fait qu'elle possède un capital intellectuel insuffisant et qu'elle accorde trop peu d'importance à la recherche et au développement. L'Asie doit fournir de plus sérieux efforts, d'abord pour créer un capital intellectuel dans la région, puis pour inverser le phénomène de l'exode des talents et, enfin, pour favoriser davantage la mobilité des ressources humaines entre les pays de l'Asie. À l'heure actuelle, il y a très peu de mobilité des personnes à l'intérieur de l'Asie pour l'enseignement et l'apprentissage (il y en a davantage dans les pays occidentaux). Il faudra peut-être faire tomber les barrières traditionnelles contre l'immigration dans le cadre du travail, car cette immigration est étroitement liée à l'immigration dans le cadre de l'enseignement et de l'apprentissage. Je crois que les pays de l'Asie doivent repenser leurs propres barrières et frontières traditionnelles. Dans une large mesure, je pense que de nombreux pays asiatiques vivent comme des étrangers dans leur propre voisinage. Il nous faut faire renaître cette époque ancienne où les grands intellectuels de Chine et les commerçants de l'Inde se déplaçaient partout dans le monde. Nous devons recréer, de manière innovante, une empreinte intellectuelle à l'intérieur de l'Asie.

Certaines initiatives s'efforcent effectivement de repérer et d'encourager le talent. À titre d'exemple, la Corée a créé ses propres établissements prestigieux grâce au programme «BK21 ${ }^{4}$. La Chine tente de faire de même avec les

4. BK21 = Brain Korea 21 Program for Leading Universities and Students. Voir le site : http://www.useoul.edu/ research/bk21-groups $(N d T)$. 
programmes « 985 » et « $221 »^{5}$. L'Inde cherche à poursuivre ses investissements dans l'enseignement supérieur. Il est important de créer du talent à l'échelle locale et de ne pas se contenter d'innovations importées d'ailleurs. Nous devons être à la pointe et investir suffisamment, sinon nous risquons de tomber dans le piège du revenu intermédiaire. Comment y parvenir ? La réponse constitue bien entendu un défi de taille.

\section{POUR UN Rôle ACCRU DES ALLIANCES RÉGIONALES}

À mon sens, cela appelle en réalité de nouveaux types d'établissements d'enseignement. Nous avons bien eu par le passé des établissements en Asie, tels que les universités de Nalanda et de Takshashila, en Inde, où convergeaient autrefois des gens de toute l'Asie pour l'enseignement universitaire qui y était dispensé, avant de repartir. Non seulement je ressens une certaine nostalgie à l'égard de ces universités, mais je pense aussi qu'il est important de créer ce que j'appelle des « établissements trans-asiatiques ». Certains instituts d'enseignement en Asie reçoivent le plein soutien de l'Association des nations de l'Asie du Sud-Est (Anase). Mais je voudrais aussi évoquer ici la naissance d'un nouvel établissement en Inde, l'université de Nalanda, recréée à Rajgir, la ville de Bouddha, où se trouvent les ruines de Nalanda. Cette université a été créée grâce à l'effort conjoint de plusieurs pays, tels que la Chine, le Japon, Singapour, l'Inde ou encore la Thaïlande. Ce sont là, je crois, les signes avant-coureurs du changement à venir : davantage de mobilité entre les pays de l'Asie, la création de talents, l'ouverture des frontières et la création d'établissements trans-asiatiques.

J'évoquerai brièvement la question de la réforme institutionnelle et des structures institutionnelles de gouvernance. L'Asie est dans une large mesure sous contrôle de l'État. Pourquoi ? Nous avons de nombreuses collaborations avec le monde occidental, également aussi des collaborations naissantes à l'échelle régionale, mais la plupart de ces collaborations se font sous l'œil très vigilant de l'État. Je crois qu'il est important de créer une atmosphère beaucoup plus libre, afin que les pays puissent se rassembler et collaborer. L'un des changements importants de notre processus de réflexion devrait concerner le rôle de l'État vis-à-vis du monde intellectuel.

Enfin, avec le développement de la mobilité et de l'urbanisation, il y aura une plus grande hétérogénéité des voisinages en Asie. Nous devons apprendre à faire face à la diversité et à l'inégalité comme jamais auparavant. Pour éviter les conflits internes, nous devons encourager les valeurs de pluralisme et d'adaptation. L'éducation est au cour de ce processus. Les jeunes doivent acquérir un nouveau sens éthique, sous-tendu par un ensemble de valeurs fondamentales qui

5. De plus amples informations sur les programmes «985» et « 221 » sont disponibles sur: http://www. chinaeducenter.com/en/cedu/ceduproject211.php (NdT). 
se déclinent selon trois axes : d'une part, le caractère inéluctable de l'interdépendance et de la collaboration (l'individualisme doit laisser place à l'interdépendance); d'autre part, la protection durable de l'environnement, appelant de nouveaux principes éthiques associant entreprise et environnementalisme; enfin, l'apprentissage de la vie en commun dans un monde qui connaît un accroissement de la diversité et de l'inégalité. Je voudrais m'intéresser plus particulièrement aux deux derniers axes. Le défi que pose la protection durable de l'environnement et des ressources implique bien plus que des solutions techniques et rationnelles aux problèmes de gaz à effet de serre ou de changement climatique. Aujourd'hui, l'éducation face à ces problèmes relève moins de la transmission de contenus liés à l'environnement, et davantage du recours aux principes qui sous-tendent les processus écologiques, afin d'aider les communautés et leurs membres à répondre au défi de la durabilité, conformément à la situation de ces communautés. Ces principes aident non seulement les individus à mieux appréhender l'environnement naturel, mais aussi à mieux se comprendre et à mieux comprendre les communautés au sein desquelles ils vivent et travaillent, et à concevoir un programme d'éducation en matière de vie durable fondé sur ces principes.

La mondialisation de l'économie et le rôle accru des alliances régionales ont pour effet de rétrécir le monde et d'ouvrir les frontières aux personnes venues de l'extérieur. Dans le scénario qui apparaît, le pluralisme et le multiculturalisme, qui devraient faire partie du processus éducatif, seront la norme et non pas l'exception. Tout pays qui tentera de percer à partir d'une vision nationaliste et culturelle étroite, agira au péril de sa propre stabilité et de sa propre progression. Les jeunes diplômés devront acquérir les bonnes compétences et adopter le bon comportement pour pouvoir vivre ensemble dans un monde caractérisé par la cohabitation de multiples perspectives religieuses, culturelles, linguistiques et idéologiques. C'est encore plus vrai en Asie qu'ailleurs dans le monde, et plus encore en Inde et en Chine à mesure que ces deux pays se mondialisent. Nous devons reconnaître le fait que pour donner au langage du développement une forme concrète et une valeur pérenne, celui-ci doit se fonder sur le partage des expériences. Il faut créer des écoles et fournir ces expériences.

Dans un tel contexte, nous espérons que « le siècle de l'Asie » adviendra.

\section{BIBLIOGRAPHIE}

ASIAN DEVELOPMENT BANK (2011): Asia 2050: Realizing the Asian Century: Executive Summary, Manille. [en ligne] http://goo.gl/fPWJ7r/

GOLDIN C. et KATZ L. (2008): The Race between Education and Technology, Cambridge, Mass. : Belknap Press of Harvard University Press. 